DOI: https://doi.org/10.11144/Javeriana.umed62-1.seda

\title{
Sedación para procedimientos en el servicio de urgencias
}

\section{Procedural Sedation in the Emergency Department}

Recibido: 10/06/2020 | Aceptado: 14/08/2020

a Autora de correspondencia: rodriguezcatalina@javeriana.edu.co

Cómo citar: Rodríguez Prada C, Peñaloza Rey NF, Parra Córdoba JF, Moreno Carrillo A. Sedación para procedimientos en el servicio de urgencias. Univ. Med. 2021;62(1). https://doi.org/10.11144/Javeriana.umed 62-1.seda

\section{RESUMEN}

En el Departamento de Urgencias, a menudo, los pacientes necesitan someterse a procedimientos diagnósticos y terapéuticos dolorosos, angustiosos o desagradables como parte de su atención. El uso de varios analgésicos, sedantes y anestésicos se ha descrito en varias guías bien referenciadas. Las diferentes opciones actuales para la administración de sedación en urgencias permiten que se realice de forma más efectiva y segura, además de que facilitan ciertos procedimientos que, de otro modo, requerirían anestesia en el quirófano. Así, se agiliza el manejo de los pacientes y disminuye su tiempo de estancia intrahospitalaria. El objetivo de esta revisión es exponer las estrategias terapéuticas tendientes a proveer el mejor manejo posible en los pacientes que requieren sedación para procedimientos no programados diagnósticos o terapéuticos en el servicio de urgencias.

Palabras clave

sedación consciente; analgesia; medicina de emergencia; servicios médicos de urgencias.

\begin{abstract}
Patients in the emergency department often need some painful, distressing, or unpleasant diagnostic and therapeutic procedures as part of their care. The use of various pain relievers, sedatives, and anesthetics has been described in several well-referenced guidelines. The different options for the administration of sedation drugs in the emergency room currently allow them to be performed more effectively and safely than in past times, in addition to facilitating the carrying out of specific procedures that would otherwise require anesthesia in the operating room, thus speeding up the management of patients and reducing the length of hospital stay of patients. The objective of this review is to present the therapeutic strategies aimed to providing the best possible management in
\end{abstract}


patients who require sedation for unscheduled diagnostic or therapeutic procedures in the emergency department.

Keywords

conscious sedation; analgesia; emergency medicine; emergency medical services.

\section{Introducción}

La sedación y la analgesia en el servicio de urgencias constituyen una práctica frecuente, cuyos objetivos son aliviar el dolor y la ansiedad, así como facilitar la realización de un procedimiento médico, a fin de mejorar la experiencia del paciente durante su atención. La sedación para procedimientos no programados involucra la administración de medicamentos sedantes o disociativos con la administración concomitante de medicamentos analgésicos o sin tal. Esta práctica requiere la presencia de un médico con experiencia y habilidades en cuidados críticos, manejo de la vía aérea, resucitación básica y avanzada, por lo cual la sedación en procedimientos no programados en urgencias es una experticia esencial en la práctica del especialista en medicina de urgencias $(1,2)$.

Teniendo en cuenta que el servicio de urgencias es un ambiente único, donde los pacientes ingresan sin ser programados, frecuentemente con problemas complejos que pueden requerir intervenciones urgentes $\mathrm{O}$ de emergencia para prevenir morbilidad $o$ mortalidad a corto, mediano y largo plazo, las cuales producen ansiedad y dolor, aun cuando necesarias para el manejo de la(s) patología(s) por la cual consulta el paciente, se hace necesario que estén claros los conceptos de sedación, al igual que conocer y saber manejar los equipos y medicamentos que se van a utilizar para realizarla $(3,4)$.

Por definición, a los pacientes que reciben sedación no se les protege de rutina las vías respiratorias con intubación endotraqueal u otros complementos de las vías respiratorias. Esto contrasta con la provisión de anestesia general, que generalmente necesita protección de las vías respiratorias $(4,5)$. Esta revisión se centra en la técnica y forma segura de llevar a cabo una sedación para procedimientos no programados en adultos en el servicio de urgencias.

\section{Definiciones}

La sedación para procedimientos es la técnica para administrar tanto medidas farmacológicas (sedantes o agentes disociativos, con analgésicos o sin estos) como no farmacológicas, para inducir un estado que permita al paciente tolerar un procedimiento no placentero al tiempo que mantiene la función cardiorrespiratoria (6).

Se desea, entonces, producir un grado de conciencia deprimido que le permita al paciente mantener un control independiente de la vía aérea y su oxigenación, preservando los reflejos protectores de la vía aérea. Los siguientes términos son importantes para entender los conceptos de esta revisión:

Ansiólisis: es un estado de aprehensión disminuida, en consideración de una situación específica en la cual el grado de conciencia del paciente no fluctúa.

Analgesia: disminución y alivio del dolor sin alteración del estado mental.

Disociación: es un estado cataléptico transitorio, caracterizado por una profunda analgesia y amnesia. Los reflejos protectores, respiración espontánea y estabilidad cardiopulmonar están preservados.

La definición de la Sociedad Americana de Anestesiología (ASA, por sus siglas en inglés) de sedación y analgesia fue creada para describir mejor los diferentes grados de sedación. Aunque esto en realidad es un continuo, la ASA lo ha dividido en cuatro subgrupos distintos: sedación mínima, sedación moderada, sedación profunda y anestesia general (tabla 1). Desde entonces se ha agregado una quinta categoría: la sedación disociativa $(7,8)$. 
Tabla 1

Definición de niveles de sedación y analgesia

\begin{tabular}{|l|l|l|l|l|l|}
\hline & $\begin{array}{c}\text { Sedación } \\
\text { mínima } \\
\text { (ansiólisis) }\end{array}$ & $\begin{array}{c}\text { Sedación } \\
\text { moderada y } \\
\text { analgesia } \\
\text { (sedación } \\
\text { consciente) }\end{array}$ & $\begin{array}{c}\text { Sedación } \\
\text { profunda y } \\
\text { analgesia }\end{array}$ & $\begin{array}{l}\text { Sedación } \\
\text { disociativa }\end{array}$ & $\begin{array}{c}\text { Anestesia } \\
\text { general }\end{array}$ \\
\hline $\begin{array}{l}\text { Capacidad de } \\
\text { respuesta }\end{array}$ & $\begin{array}{l}\text { Respuesta } \\
\text { normal a la } \\
\text { estimulación } \\
\text { verbal }\end{array}$ & $\begin{array}{l}\text { Respuesta } \\
\text { intencional a } \\
\text { la } \\
\text { estimulación } \\
\text { verbal o táctil }\end{array}$ & $\begin{array}{l}\text { Respuesta } \\
\text { intencional a } \\
\text { la } \\
\text { estimulación } \\
\text { repetida o } \\
\text { dolorosa }\end{array}$ & $\begin{array}{l}\text { Puede ser } \\
\text { inadecuada. } \\
\text { Inarusable, } \\
\text { incluso con } \\
\text { estimulo } \\
\text { doloroso }\end{array}$ & $\begin{array}{l}\text { Puede ser } \\
\text { inadecuada. } \\
\text { Inarusable, } \\
\text { incluso con } \\
\text { estímulo } \\
\text { doloroso }\end{array}$ \\
\hline Vía aérea & No afectada & $\begin{array}{l}\text { No se } \\
\text { requere } \\
\text { intervención }\end{array}$ & $\begin{array}{l}\text { Se puede } \\
\text { requerir } \\
\text { intervención }\end{array}$ & $\begin{array}{l}\text { Puede } \\
\text { requerir } \\
\text { intervención }\end{array}$ & $\begin{array}{l}\text { La intervención, } \\
\text { a menudo, se } \\
\text { requiere }\end{array}$ \\
\hline $\begin{array}{l}\text { Ventilación } \\
\text { espontánea }\end{array}$ & No afectada & Adecuada & $\begin{array}{l}\text { Puede ser } \\
\text { inadecuada }\end{array}$ & Adecuada & $\begin{array}{l}\text { Frecuentemente } \\
\text { inadecuada }\end{array}$ \\
\hline $\begin{array}{l}\text { Función } \\
\text { cardiovascular }\end{array}$ & No afectada & $\begin{array}{l}\text { Generalmente } \\
\text { mantenida }\end{array}$ & $\begin{array}{l}\text { Generalmente } \\
\text { mantenida }\end{array}$ & Elevada & $\begin{array}{l}\text { Puede estar } \\
\text { deteriorada }\end{array}$ \\
\hline
\end{tabular}

Fuente: adaptado de (6).

\section{Niveles de sedación}

Sedación mínima (ansiólisis). Se describe la alteración del estado de conciencia y funciones cognitivas, el cual es similar a la basal. En este estado, el paciente preserva los reflejos protectores de la vía aérea, de la ventilación espontánea y del estado cardiovascular, con respuesta normal al estímulo verbal. Es la adecuado para procedimientos menores. En este nivel, el paciente tiene alterada su función cognitiva y coordinación, pero responde fácilmente a comandos verbales y está tranquilo.

Sedación moderada (sedación consciente). Se entiende por aquel estado de conciencia deprimido en el cual el paciente responde a estímulos verbales solos o con acompañamiento de estímulo táctil, preservando en igual medida la respiración espontánea y sin requerimiento de intervención en la vía aérea con estabilidad hemodinámica. En este estado, los pacientes frecuentemente presentas ptosis de los párpados, bradilalia y discurso similar a la disartria. Con frecuencia en este estado se produce la amnesia.

Sedación profunda. En este estado, el paciente no es fácilmente alterable, responde ante estímulos fuertes o dolorosos. Podría estar afectada la capacidad para mantener una ventilación espontánea y el estado cardiovascular, y llegar a requerir soporte ventilatorio y hemodinámico.

Sedación disociativa. Es un estado transitorio cataléptico con analgesia profunda y amnesia; pero sin alteración de los reflejos protectores de la vía aérea, la respiración espontánea o la estabilidad cardiopulmonar. Facilita la realización de procedimientos moderados a severamente dolorosos, así como procedimientos que requieran inmovilización o no haya cooperación del paciente. Este tipo de sedación se logra específicamente con la ketamina.

Anestesia general. Es una pérdida de conciencia inducida por medicamentos durante la cual los pacientes no pueden despertarse, incluso con estimulación dolorosa. La capacidad de mantener la función ventilatoria de forma independiente, a menudo, se ve afectada, por lo que requiere asistencia para mantener una vía aérea permeable. La función cardiovascular puede verse afectada.

La progresión de la sedación mínima a la anestesia general es un continuo dinámico que carece de una separación clara entre las etapas. La transición de una sedación a la siguiente, a menudo, es difícil de predecir y varía de un paciente a otro. El continuo de sedación no es específico del fármaco y se pueden alcanzar todos los niveles de sedación de leve a anestesia general con prácticamente todos los medicamentos. Debido a esto, se recomienda que los médicos de urgencias que administren sedación sean competentes en las habilidades requeridas para tratar a pacientes que tienen, al menos, un nivel mayor que el nivel de sedación deseado y las complicaciones que se puedan presentar (1).

\section{Manejo}

Administrar sedación en el servicio de urgencias para un procedimiento, implica considerar la condición clínica del paciente y sus comorbilidades, la urgencia, la profundidad de la sedación requerida y la duración necesaria. Las decisiones respecto al plan de sedación del procedimiento deben equilibrarse entre la urgencia y la probabilidad de complicaciones. Estas decisiones deben tomarse con el paciente o los cuidadores cuando sea posible, con el consentimiento apropiado (9).

Una herramienta común y útil para evaluar la idoneidad del paciente en cuanto a la sedación es el sistema de clasificación del estado físico 
de la ASA (tabla 2) (10). El riesgo de una complicación significativa con sedación en el servicio de urgencias en las clases I y II de la ASA suelen ser menores al $5 \%$ y aumenta a medida que incrementa la clasificación ASA, aunque también se ha informado la sedación de procedimientos segura en el servicio de urgencias para las clases III y IV $(11,12)$.

Los procedimientos no programado en urgencias que deben realizarse dentro del contexto de sedación son $(13,14,15,16,17)$ :

Administración de sedación para procedimientos.

Reducción de fracturas o luxaciones.

Incisión y drenaje de abscesos.

Cardioversión eléctrica.

Toracostomía cerrada.

Punción lumbar.

Sutura de heridas complejas.

Toma de estudios imagenológicos.

No aplica para:

Sedación en pacientes críticamente enfermos.

Sedación en cuidados paliativos.

Procedimientos que requieran anestesia general.

Protocolo para secuencia de intubación.

\section{Tabla 2}

Sistema de clasificación de la Sociedad Americana de Anestesiólogos

\begin{tabular}{|l|l|l|}
\hline Clase & \multicolumn{1}{|c|}{ Descripción } & \multicolumn{1}{c|}{ Ejemplo } \\
\hline I & Saludable & - isté \\
\hline II & Enfermedad sistémica leve & $\begin{array}{l}\text { Asma leve, embarazo, obesidad, diabetes } \\
\text { controlada }\end{array}$ \\
\hline III & Enfermedad sistémica grave & $\begin{array}{l}\text { Neumonia, asma moderada a grave, } \\
\text { enfermedad renal en etapa terminal }\end{array}$ \\
\hline IV & $\begin{array}{l}\text { Enfermedad sistémica grave } \\
\text { que es una amenaza constante } \\
\text { para la vida }\end{array}$ & $\begin{array}{l}\text { Septicemia, disfunción cardiaca grave, } \\
\text { coagulación intravascular diseminada }\end{array}$ \\
\hline V & $\begin{array}{l}\text { Moribundo y no se espera que } \\
\text { sobreviva sin cirugía }\end{array}$ & $\begin{array}{l}\text { Trauma masivo, hemorragia intracraneal con } \\
\text { efecto de masa, intestino isquémico con } \\
\text { disfunción de múltiples órganos }\end{array}$ \\
\hline VI & $\begin{array}{l}\text { Declarado muerte cerebral } \\
\text { cuyos órganos se están } \\
\text { extrayendo }\end{array}$ & \\
\hline
\end{tabular}

Fuente: tomado y adaptado de (10).

\section{Personal, equipo e insumos necesarios}

Se requiere, como mínimo, la disposición de un médico especialista en medicina de urgencias, distinto al médico que realizará el procedimiento, con entrenamiento y familiarizado con la sedación y el manejo de sus posibles complicaciones, y una persona profesional en enfermería para asistir al personal médico. Es preciso que estos profesionales estén entrenados en soporte vital básico y avanzado, así como que entiendan las características farmacológicas, mecanismos de acción y efectos adversos de los medicamentos que se van a usar (18).

Debe contarse con profesionales entrenados para la valoración previa, durante la realización de la sedación y después de esta, con la capacidad para establecer la pertinencia y la oportunidad de la sedación, en que valoren la historia clínica, el examen físico dirigido y la valoración del riesgo, así como con la capacidad de detectar, monitorizar y tratar las complicaciones esperables de este procedimiento (16).

Debe realizarse en un entorno con disponibilidad inmediata a equipos de monitoreo y reanimación, por lo que para todo paciente que se va a someter a una sedación para procedimientos en urgencias se debe contar con $(2,11,19,20,21,22)$ :

Equipo de succión.

Fuente de oxígeno.

Equipo de vía aérea.

Equipo de acceso vascular.

Medicamentos.

Carro de paro.

Equipo de monitorización no invasiva.

\section{Preparación}

Antes de iniciar el proceso de sedación se debe valorar al paciente de forma adecuada y tener en cuenta varias consideraciones, por ejemplo: verificar indicadores de ventilación difícil, vía aérea difícil o colocación de dispositivo supraglótico difícil, para así mejorar la atención y la preparación ante posibles complicaciones, o definir si es necesario diferir o realizar el procedimiento en otro ámbito, como las salas de cirugías (23).

Así mismo, es preciso evaluar las complicaciones más probables que tiene el paciente según sus comorbilidades y determinar el equipo necesario para asegurar la vía aérea en caso de ser necesario, alergias, uso de sustancias 
psicoactivas, alcohol y un adecuado examen físico general.

No es recomendable en el escenario de urgencias retrasar la sedación y el procedimiento que necesita el paciente si no se cumplen con los tiempos de ayuno; sin embargo, en caso tal que los riesgos se puedan disminuir con la posibilidad de diferir la sedación hasta tener tiempos de ayuno seguros, las guías de sedación por fuera del ámbito del servicio de urgencias y emergencias plantean mantener un ayuno mínimo de 2 horas para líquidos claros (agua, té o jugo en agua) y 6 horas para sólidos, que son protocolos usados generalmente en el contexto de salas de cirugía, donde usualmente el paciente requiere sedación profunda $(24,25,26)$.

Múltiples revisiones sistemáticas no han podido definir un tiempo de ayuno específico que disminuya los eventos de aspiración o complicaciones de la sedación; adicionalmente, en la literatura no se ha evidenciado que el no presentar ayuno aumente la frecuencia e incidencia de la emesis durante la sedación para procedimientos en el servicio de urgencias; pero es importante tener claridad que esto puede llevar a un riesgo potencial y que se debe sopesar este contra el beneficio y la emergencia del procedimiento que requiere el paciente. Por ello, recomendamos siempre preguntar respecto al ayuno, para identificar posibles riesgos y dejar en consideración al médico tratante la realización diferida o no de la sedación $(2,27,28)$.

Se debe trasladar al paciente al área de reanimación o a un área que disponga de todos los equipos necesarios para el manejo de monitorización, vigilancia clínica y complicaciones. Respecto a la monitorización no invasiva del paciente, esta debe contar con equipos para la toma de tensión arterial, frecuencia cardiaca, frecuencia respiratoria, saturación de oxígeno, electrocardiograma por visoscopio y capnografía, en caso de tenerla disponible (4).

Una vez se haya realizado la valoración integral al paciente, se procederá a decidir cuál(es) es(son) los medicamentos necesarios para llevar a cabo la sedación, teniendo en cuenta los objetivos ideales de realizarla y lograr la sedación deseada $(23,29)$ :

1. Dar tranquilidad sobre el procedimiento.

2. Obtener la cooperación del paciente.

3. Inducir inconciencia y amnesia.

4. Disminuir el disconfort y dolor.

5. Mantener la seguridad del paciente.

\section{Medicamentos}

Los medicamentos más utilizados para la sedación son los siguientes (tabla 3).

Tabla 3

Medicamentos usados para la sedación

\begin{tabular}{|c|c|c|c|c|c|}
\hline Medicamento & $\begin{array}{c}\text { Mecanismo de } \\
\text { acción }\end{array}$ & Metabolismo & $\begin{array}{c}\text { Tiempo de } \\
\text { latencia/duración }\end{array}$ & Dosis & Efectos adversos \\
\hline Midazolam & $\begin{array}{l}\text { Benzodiacepina, } \\
\text { agonista en } \\
\text { receptores } \\
\text { GABA-A. }\end{array}$ & $\begin{array}{l}\text { Hepático } \\
\text { (CYP3A4). }\end{array}$ & \begin{tabular}{|l} 
Tiempo \\
latencia: 1-2 \\
minutos por IV. \\
Duración: $30-60$ \\
minutos.
\end{tabular} & $\begin{array}{l}\text { La dosis para } \\
\text { adultos es de } \\
0,02 \text { a } 0,03 \\
\mathrm{mg} / \mathrm{kg} \text { y } \\
\text { titular. } \\
\text { Bolo inicial } \\
\text { máximo de } \\
2,5 \mathrm{mg} \text { IV. } \\
\end{array}$ & \begin{tabular}{|l|} 
Hipotensión, \\
hipoxemia, e \\
infrecuentes \\
como depresión \\
del SNC, \\
cefalea, \\
náuseas, emesis \\
e hipo. \\
\end{tabular} \\
\hline Propofol & $\begin{array}{l}\text { Agente } \\
\text { alquifenol, } \\
\text { agonista en } \\
\text { receptor GABA-A } \\
\text { e inhibidor de } \\
\text { receptores } \\
\text { NMDA. }\end{array}$ & \begin{tabular}{|l} 
Conjungación \\
hepática a \\
compuesto \\
incativo.
\end{tabular} & \begin{tabular}{|l} 
Tiempo \\
latencia: menor \\
a 1 minuto. \\
Duración: 8 a 10 \\
minutos.
\end{tabular} & $\begin{array}{l}\text { Bolos de } 0,5 \\
\mathrm{mg} / \mathrm{kg} \text { IV y } \\
\text { titular cada 1 a } \\
3 \text { minutos con } \\
\text { dosis } \\
\text { adicionales } \\
\text { entre } 0,25 \text { y } \\
0,5 \mathrm{mg} / \mathrm{kg} \\
\text { hasta en nivel } \\
\text { de sedación } \\
\text { deseado. }\end{array}$ & $\begin{array}{l}\text { Dolor, } \\
\text { depresión } \\
\text { respiratoria } \\
\text { profunda, } \\
\text { apnea, } \\
\text { disminución del } \\
\text { gasto cardiaco o } \\
\text { hipotensión. }\end{array}$ \\
\hline Ketamina & $\begin{array}{l}\text { Agente } \\
\text { disociativo, } \\
\text { mecanismo de } \\
\text { acción en } \\
\text { receptores } \\
\text { NMDA, opioides, } \\
\text { muscarinicos, } \\
\text { GABA y } \\
\text { monoaminérgicos. }\end{array}$ & $\begin{array}{l}\text { Hepático por } \\
\text { demetilación } \\
\text { de la } \\
\text { ketamina. }\end{array}$ & \begin{tabular}{|l} 
Tiempo \\
latencia: menor \\
a 1-5 minutos. \\
Duración: $15-30$ \\
minutos.
\end{tabular} & $\begin{array}{l}\text { Dosis inicial } \\
\text { de } 1-2 \mathrm{mg} / \mathrm{kg} \\
\text { IV o } 4-5 \\
\mathrm{mg} / \mathrm{kg} \text { IM } \\
\text { puede requerir } \\
\text { dosis } \\
\text { adicionales } \\
\text { cada } 15 \text { a } 20 \\
\text { minutos de } \\
0,25 \text { a } 0,5 \\
\mathrm{mg} / \mathrm{kg} \mathrm{IV} . \\
\end{array}$ & \begin{tabular}{|l} 
Sueños vividos \\
no placenteros \\
o alucinaciones, \\
agitación \\
psicomotora, \\
apnea \\
transitoria y \\
laringoespasmo, \\
náusea y \\
emesis. \\
\end{tabular} \\
\hline Etomidato & $\begin{array}{l}\text { Derivado del } \\
\text { imidazol, agonista } \\
\text { en receptores } \\
\text { GABA-A. }\end{array}$ & $\begin{array}{l}\text { En sangre por } \\
\text { esterasas } \\
\text { plasmáticas y } \\
\text { hepática. }\end{array}$ & \begin{tabular}{|l} 
Tiempo \\
latencia: 45 \\
segundos. \\
Duración: 5-10 \\
minutos.
\end{tabular} & $\begin{array}{l}\text { Dosis inicial } \\
\text { de } 0,1 \mathrm{mg} / \mathrm{kg} \\
\text { con bolos } \\
\text { adicionales de } \\
0,05 \text { a } 0,1 \\
\mathrm{mg} / \mathrm{kg} \text { cada } 2 \\
\text { a } 3 \text { minutos } \\
\text { hasta nivel de } \\
\text { sedación } \\
\text { deseado. } \\
\end{array}$ & \begin{tabular}{|l} 
Dolor, apnea, \\
depresión \\
respiratoria, \\
mioclonias, \\
náuseas, emesis \\
y supresión \\
adrenal.
\end{tabular} \\
\hline Fentanilo & $\begin{array}{l}\text { Actúan en los } \\
\text { recptores opiodes } \\
\mu(\mu 1 \text { y } \mu 2), \kappa, \delta \text {, } \\
\sigma, y \varepsilon \text {. }\end{array}$ & \begin{tabular}{|l} 
Hepático \\
(CYP3A4).
\end{tabular} & \begin{tabular}{|l} 
Tiempo de \\
latencia: $1-2$ \\
minutos. \\
Duración: $30-40$ \\
minutos.
\end{tabular} & $\begin{array}{l}1-3 \mu \mathrm{g} / \mathrm{kg} \\
\text { previo a la } \\
\text { administración } \\
\text { del } \\
\text { medicamento } \\
\text { sedante. }\end{array}$ & \begin{tabular}{|l|} 
Depresión \\
respiratoria, \\
náuseas, \\
emesis, \\
hipotensión, \\
bradicardia, \\
tórax en leño y \\
el espasmo \\
glótico. \\
\end{tabular} \\
\hline
\end{tabular}

\section{Midazolam}

Es una benzodiacepina con propiedades ansiolíticas, amnésicas, sedantes, hipnóticas y anticonvulsivas, que no presenta efectos analgésicos, por lo que se recomienda el uso concomitante de opioides. Tienen un mecanismo de acción agonista en receptores (ácido $\gamma$ amino butírico) GABA-A. Las acciones farmacológicas 
de este medicamento son afectadas por la obesidad, la edad, la hepatopatía y la condición crítica. Debido a su característica lipofílica a $\mathrm{pH}$ fisiológico, se va a distribuir preferiblemente a los adipocitos, lo que implica una eliminación de la vida media prolongada (6).

Se puede administrar vía endovenosa (IV), intramuscular (IM) u oral (VO), aun cuando la más frecuente es la IV. Su tiempo de latencia dependerá de la vía de administración, que es de 1 a 2 minutos por IV, con una duración de 30 a 60 minutos. La dosis para adulto es de 0,02 a 0,03 $\mathrm{mg} / \mathrm{kg}$. Se prefiere iniciar con la dosis más baja e ir titulando según el efecto deseado y la respuesta del paciente. El bolo inicial máximo es de $2,5 \mathrm{mg}$ IV $(6,30)$.

Puede presentar efectos adversos dosis dependiente, siendo estos la hipotensión y la hipoxemia, y aunque son infrecuentes se asocian con una depresión del sistema nervioso central. Otros efectos adversos son cefalea, náuseas, emesis e hipo. Los pacientes obesos, ancianos, con enfermedad renal o hepática son la población con mayor riesgo a presentarlos con mayor probabilidad de depresión respiratoria cuando lo asociamos a un opioide (30).

\section{Propofol}

Es un agente alquifenol de acción ultracorta, altamente liposoluble, con una composición del medicamento con aceite de soya y fosfolípidos de huevo como emulsificador, con el potencial de ocasionar reacciones alérgicas a estos componentes y presentar dolor a la administración. Es estable a temperatura ambiente, sin sensibilidad a la luz. Cuenta con un mecanismo de acción de agonista en receptor GABA-A e inhibidor de receptores (n-metil-daspartato) NMDA (6).

El propofol provee efectos sedantes, amnésicos y antieméticos. Con la administración IV su tiempo de latencia es menor a 1 minuto y su duración es de 8 a 10 minutos a dosis de 0,5 $\mathrm{mg} / \mathrm{kg}$. Este medicamento no presenta efectos analgésicos, por lo cual se recomienda asociarlo con un opioide en procedimientos dolorosos $(30,31)$.

Puede producir depresión respiratoria profunda, apnea, disminución del gasto cardiaco e hipotensión, por lo que se recomienda usarlo a dosis bajas, en especial en pacientes adultos mayores y pacientes hipovolémicos. Se ha asociado con que el paciente puede requerir soporte con ventilación positiva por depresión respiratoria (27). Se recomienda usar bolos de $0,5 \mathrm{mg} / \mathrm{kg}$ IV y titularlo cada 1 a 3 minutos con dosis adicionales entre $0,25-0,5 \mathrm{mg} / \mathrm{kg}$ hasta que se llegue a la sedación deseada $(6,32)$.

\section{Ketamina}

Es un agente disociativo que produce un estado catatónico, analgesia y amnesia. Su mecanismo de acción se da con receptores NMDA, opioides, muscarínicos, GABA y monoaminérgicos. Crea una disrupción entre el sistema talamocortical y el límbico, por lo cual los pacientes frecuentemente presentan nistagmos y movimientos anormales de las extremidades no relacionados con estímulos dolorosos. La ventaja de la ketamina es su propiedad analgésica adicional, con poca depresión respiratoria. Los reflejos protectores de la vía aérea son preservados, con un efecto hemodinámico de taquicardia e hipertensión por inhibición de la recaptación de catecolaminas y su efecto broncodilatador. Se puede administrar por vía IV o IM, con un tiempo de latencia menor a 1 minuto, y de 5 minutos, según la vía respectivamente. La duración de su efecto está entre 15 y 30 minutos $(33,34,35)$.

Dentro de los efectos adversos, es posible que el paciente tenga un despertar en el que refiera sueños vividos no placenteros o alucinaciones, agitación psicomotora, por lo cual se ha asociado al uso concomitante de midazolam, que ayuda a disminuir la incidencia de estos síntomas, que aunque benignos y autolimitados son incómodos para el paciente. Con menor frecuencia se ha documentado apnea transitoria y laringoespasmo asociado a una administración del medicamento rápida. Otro efecto adverso que vale la pena 
mencionar es la náusea y el vómito, que se puede manejar con ondansetrón $(36,37)$.

Se recomienda evitar el uso de ketamina en pacientes con enfermedad coronaria o arterial, por sus efectos catecolaminérgicos, hipertensión intracraneal y síndrome convulsivo (32). Se plantea una dosis inicial de $1.2 \mathrm{mg} / \mathrm{kg}$ IV o $4-5 \mathrm{mg} / \mathrm{kg} \mathrm{IM}$, aun cuando es posible que ser requieran dosis adicionales cada 15 a 20 minutos de 0,25 a $0,5 \mathrm{mg} / \mathrm{kg}$ IV $(6,30)$.

\section{Etomidato}

Es un derivado del imidazol, con un mecanismo de acción en receptores GABA-A que produce hipnosis sin efecto analgésico, con acción corta. Es de administración IV con un tiempo de latencia de 45 segundos y duración de 5-10 minutos, con mínimos efectos respiratorios y cardiovasculares $(6,38)$.

Entre sus efectos adversos están apnea, depresión respiratoria, mioclonías, náuseas, vómito y supresión adrenal. Es de tener en cuenta que las mioclonías pueden llegar a ser tan importantes que impidan el procedimiento para el cual se administró la sedación. También produce dolor cuando se administra por una vena periférica, lo cual se puede manejar al igual que con el propofol: una dosis previa de lidocaína o fentanilo $(39,40)$. Se recomienda una dosis inicial de $0,1 \mathrm{mg} / \mathrm{kg}$ con bolos adicionales de 0,05 a 0,1 $\mathrm{mg} / \mathrm{kg}$ hasta lograr el nivel de sedación deseado cada 2 a 3 minutos $(6,30)$.

\section{Fentanilo}

El uso de opioides es altamente utilizado en asociación con medicamentos sedantes, debido a que estos últimos no presentan efecto analgésico. Los opioides, raramente, presenta efectos sedantes como monoterapia; sin embargo, al realizar la combinación de medicamentos se debe valorar continuamente el riesgo de depresión respiratoria. El fentanilo presenta múltiples ventajas, como que su inicio de acción y duración es corta, no liberan histamina y tienen un adecuado perfil cardiovascular. Es de administración IV con tiempo de latencia de 1-2 minutos y duración de 30-40 minutos (41).

Dentro de los efectos adversos de este medicamento se encuentran: depresión respiratoria (dosis dependiente o cuando se asocia con otros medicamentos o previo consumo de alcohol), náuseas, emesis, hipotensión y bradicardia. Cabe resaltar el efecto de tórax en leño y el espasmo glótico, que se asocian a la velocidad de administración del medicamento y la dosis (42). Se recomienda el uso de 1-3 $\mu \mathrm{g} /$ $\mathrm{kg}$ previo a la administración del medicamento sedante. Es preciso tener en cuenta que en pacientes ancianos se debe utilizar la dosis mínima $(6,30)$.

\section{Medicamentos de rescate/antídotos}

En ocasiones se presentan escenarios en los cuales se genera una sedación más profunda de la deseada o alteraciones del sistema respiratorio, como es la depresión respiratoria y la apnea, que precisan intervenciones inmediatas, como asegurar la vía aérea, usar oxígeno suplementario o un dispositivo válvula-máscara bolsa, en el cual se requieren otros medicamentos para revertir el efecto farmacológico de los medicamentos usados. No se recomiendan estos medicamentos para devolver el efecto sedante una vez se haya llevado a cabo el procedimiento (6).

\section{Naloxona}

Es un antagonista competidor de los receptores de opioides y es usada en la depresión respiratoria secundaria a estos medicamentos. No se recomienda en escenarios de tórax en leño. Tiene un inicio de acción rápida con una duración del efecto de entre 15 y 30 minutos. Su administración puede ser IV, IM o endotraqueal, y se recomienda titular su dosis con bolos de 0,1-0,2 mg IV cada 1 a 2 minutos. Dependiendo de la vida media del opioide administrado, se pueden requerir dosis adicionales de naloxona o infusión (6).

Es necesario tener en cuenta posibles efectos adversos, como lo son la elevación de la 
presión arterial, edema pulmonar, convulsiones y arritmias cardiacas (30).

\section{Flumazenilo}

Es un antagonista competitivo de las benzodiacepinas, el cual, a pesar de revertir el efecto sedante, no revierte la depresión respiratoria. Tiene un tiempo de latencia de 1-2 minutos con una duración de 30-60 minutos (6). Se recomienda titular su dosis en bolos de 0,1-0,2 mg cada minuto, con una dosis máxima de $1 \mathrm{mg}$ (6).

\section{Recomendaciones}

Procedimientos no dolorosos (imageneología). Se recomienda el uso de midazolam según su perfil cardiovascular, como alternativas se recomienda el propofol $(43,44)$.

Procedimiento doloroso leve con alta ansiedad. Como lo son la realización de implantación de catéter venoso central o punción lumbar, se recomienda el uso de midazolam, ketamina o propofol, asociado con un analgésico ya sea local o sistémico $(43,45)$.

Procedimiento moderado a severamente doloroso y alta ansiedad. Como lo son la realización de reducción de fracturas o luxaciones, drenaje de abscesos, desbridamiento de quemaduras, cardioversión o toracostomía cerrada. Se recomienda el uso de propofol, etomidato o midazolam asociado con fentanilo o el uso de ketamina (43).

Monitorización y recuperación de la sedación. La monitorización del paciente se debe continuar una vez haya acabado el procedimiento hasta que el paciente esté despierto espontáneamente y sea capaz de realizar sus funciones basales de forma independiente. Para dar por finalizada la monitorización de los efectos de la sedación y darle egreso al paciente de la sala de reanimación y continuar su atención médica, ya sea en piso de hospitalización o ambulatoriamente, se requiere que el paciente sea capaz de seguir órdenes, hablar claramente y deambular sin ayuda, siempre y cuando estas condiciones sean las previas a la sedación. Además, presentar signos vitales normales y tener un adecuado control del dolor y de otros síntomas como náuseas y vómito en caso tal de presentarse (2).

\section{Técnica general}

1. Ofrecerle al paciente las opciones de sedación, ansiólisis o analgesia vs. anestesia local y tomar la decisión según las preferencias del paciente y el criterio médico.

2. Un especialista en medicina de urgencias, diferente al médico que realiza el procedimiento que genera la necesidad de sedoanalgesia, debe hacer la sedación y la valoración previa.

3. El médico que realiza la sedación debe dedicarse exclusivamente a esto y no estar involucrado en otros procedimientos.

4. Interrogar al paciente y realizarle un examen físico para valorar los riesgos de vía aérea difícil, ventilación difícil y de complicaciones. Además, se debe le explicar objetivos, riesgos, beneficios y limitaciones de la sedación.

5. Resolver expectativas y dudas del paciente en cuanto al procedimiento.

6. Obtener un consentimiento informado previo a la administración de la sedación.

7. Trasladar al paciente al área de reanimación (o área que asegure todos los elementos y equipos necesarios) y mantener como mínimo una monitorización no invasiva de los signos vitales, antes de la aplicación de los medicamentos y la realización del procedimiento, durante este y después.

8. Administrarle a todos los pacientes sometidos a sedación suplencia de oxígeno, con el fin de evitar episodios de hipoxemia durante el procedimiento. Puede ser por cánula nasal o el dispositivo que se considere.

9. Si la sedación se va a realizar en tomografía o resonancia, trasladar al 
paciente con el personal de enfermería y el especialista en medicina de urgencias que va a realizar la sedación. El paciente debe permanecer todo el tiempo con monitorización no invasiva de signos vitales (presión arterial, frecuencia cardiaca y saturación de oxígeno). Se deben tener a la mano todos los implementos necesarios para la administración de medicamentos y resolución de complicaciones, en caso de presentarse. El personal de enfermería y médico deben acompañar y vigilar el paciente durante el tiempo que se demore la toma de imágenes y posterior a esto trasladar al paciente a sala de reanimación u observación/ expansión (según su ubicación previa y estado hemodinámico y neurológico del paciente posterior a la sedación), donde el paciente va a permanecer monitorizado hasta la recuperación de la sedación.

10. Administrar los medicamentos sedoanalgésicos según su perfil cardiovascular y comorbilidades (tabla 3).

11. Evaluar continuamente las complicaciones que se pueden presentar con la sedación (depresión respiratoria, apnea, obstrucción de la vía aérea, aspiración, etc.).

12. Realizar maniobras de aseguramiento de la vía aérea con dispositivos definitivos (intubación orotraqueal o cricotiroidotomía) en caso tal de requerirlo, según las complicaciones que se presenten y el estado hemodinámico y ventilatorio del paciente.

13. Continuar con la monitorización y vigilancia clínica hasta que se el paciente se recupere de la sedación, cumpliendo las siguientes características:

a) Se mantenga en un estado de alerta igual al previo a la sedación y este sea espontáneo. b) Mantenga sus funciones cognitivas basales.

c) Lleve a cabo sus actividades básicas basales.

d) Siga órdenes según sus comorbilidad y funcionalidad basal (por ejemplo, deambular sin ayuda, si previamente no requería ninguna asistencia).

e) Normalización de los signos vitales.

f) Adecuado control del dolor.

14. Mantener siempre un registro en la historia clínica de la sedación que contenga signos vitales previos, durante y posrecuperación; tipo de medicamento y dosis utilizada; además de la duración total de la sedación.

\section{Complicaciones}

Debido a que a los pacientes se les administran medicamentos que suprimen la conciencia, el médico debe tener experiencia en el manejo de posibles complicaciones. La más grave es la insuficiencia respiratoria, por obstrucción o hipoventilación de las vías respiratorias. Las habilidades avanzadas de manejo de las vías respiratorias son un requisito obligatorio para una sedación.

Todos los medicamentos y sus vías de administración se han relacionado con efectos adversos. Se debe evitar la combinación de tres o más agentes farmacológicos, por el aumento de la incidencia de efectos adversos. Los previsibles más comunes son $(32,37,43,46,47)$ :

Hipoxia: es el evento adverso más común. Se ha reportado con el uso de todos los medicamentos nombrados en este artículo, con una incidencia del $25 \%$, con midazolam, fentanilo y propofol; del $3 \%$ al $15 \%$, con el uso de etomidato, y en menor proporción, con ketamina. Se debe asegurar la suplencia de oxígeno por el dispositivo adecuado para garantizar niveles óptimos de saturación de oxígeno hasta la colocación de una vía aérea definitiva, si se requiere. 
Vómito: es el segundo evento adverso más común que se presenta, con una incidencia de 16,4 por cada 1000 sedaciones que se realizan. Su manejo es sintomático.

Laringoespasmo: descrito en la literatura con una incidencia del 0,4\% con el uso de ketamina, generalmente trasitorio.

Apnea: se presenta más con el uso de midazolam, con una incidencia de 51,4 por cada 1000 sedaciones. Su manejo debe ser inmediato con el uso de medicamentos de rescate/antídotos y control de la vía aérea.

Aspiración pulmonar clínicamente aparente.

Sedación inadecuada: se deben optimizar las dosis requeridas, según las pautas que se dan para el medicamento elegido hasta lograr el nivel deseado de sedación.

Sedación prolongada: en caso de que sea necesario y apropiado, se puede optar por el uso de medicamentos de rescate y siempre analizar causas secundarias por las cuales se está generando en el paciente una sedación prolongada. En estos casos, el paciente siempre debe permanecer monitorizado.

Agitación paradójica: esta se presenta con gran asociación con el uso de ketamina. Su manejo es sintomático y de vigilancia mientras dura el efecto del medicamento.

\section{Conclusiones}

La sedación no programada para procedimientos en los servicios de urgencias, le permiten al médico especialista en urgencias ejecutar el proceso de manera segura y eficiente, con el propósito de facilitarlos en el centro de emergencias. Las pautas de sedación varían según la institución; pero generalmente las establecen los comités hospitalarios y se basan en los estándares establecidos por las sociedades médicas, como el Colegio Americano de Médicos de Emergencia.

La recopilación de la historia clínica, el examen físico del paciente previo a la sedación y el análisis de los datos fisiológicos cuando se administra la sedación son de vital importancia para obtener los mejores resultados de los pacientes sometidos a sedación, y ha mostrado que se evitan desenlaces adversos (figura 1).

Si bien la práctica segura y la conciencia de la sedación y la analgesia en los procedimientos son necesarios, lo primordial es que el personal tenga la mejor capacitación para proveer un procedimiento seguro y eficaz $(9,30,48,49,50)$.

Figura 1.

Sedación en el servicio de urgencias

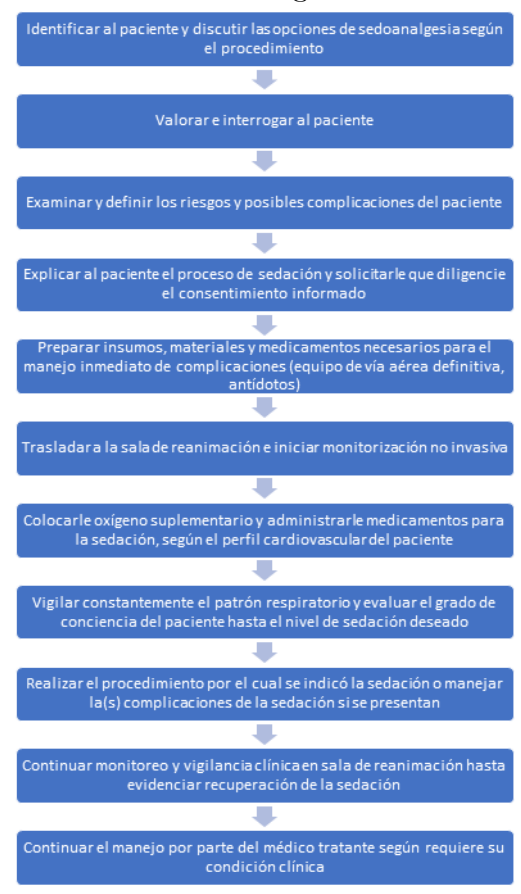

\section{Conflicto de intereses}

Los autores no reportan conflictos de intereses en este trabajo.

\section{Referencias}

1. Godwin SA, Burton JH, Gerardo CJ, Hatten BW, MacE SE, Silvers SM, et al. Clinical policy: procedural sedation and analgesia in the emergency department. Ann Emerg Med. 2014;63(2):247-58

2. Green SM, Roback MG, Krauss BS, Miner JR, Schneider S, Kivela PD, et al. Unscheduled procedural sedation: a multidisciplinary consensus practice guideline. Ann Emerg 
Med. 2019;73(5):e51-65. https://doi.org/10 .1016/j.annemergmed.2019.02.022

3. Freeston JA, Leal A, Gray A. Procedural sedation and recall in the emergency department: the relationship between depth of sedation and patient recall and satisfaction (a pilot study). Emerg Med J. 2012;29(8):670-2.

4. American Society of Anesthesiologists. Practice guidelines for sedation and analgesia by non-anesthesiologists. Anesthesiology. 2002;96:1004-17.

5. Green SM, Roback MG, Krauss BS. The newest threat to emergency department procedural sedation. Ann Emerg Med. 2018;72(2):115-9. https://doi.org/10.1016/j .annemergmed.2017.12.008

6. Godwin SA. Procedural sedation and analgesia. En: Rosen's emergency medicine: concepts and clinical practice. 9. $\stackrel{\mathrm{a}}{\mathrm{ed}}$. New York: Elsevier; 2017. p. 52-61.

7. Anesthesiologists A society for. Continuum of depth of sedation: definition of general anesthesia and levels of sedation/analgesia. Monit Anesth Care. 2019;1-2.

8. Green S, Manson K. Reformulation of the sedation continuum steven. JAMA. 2010;303 (9):876-7.

9. Bader AM, Pothier MM. Out-of-operating room procedures: preprocedure assessment. Anesthesiol Clin. 2009 Mar;27 (1):121-6.

10. American Society of Anesthesiologists. ASA Physical Status Classification System. 2019 oct 23 [internet]. Disponible en: https://www.asahq.org/standards-and-g uidelines/asa-physical-status-classificationsystem

11. Miner JR, Martel ML, Meyer M, Reardon R, Biros MH. Procedural sedation of critically ill patients in the emergency department. Acad Emerg Med. 2005;12(2):124-8.

12. Weaver CS, Hauter WE, Brizendine EJ, Cordell WH. Emergency department procedural sedation with propofol: is it safe? J Emerg Med. 2007 Nov;33(4):355-61.

13. Weaver CS, Terrell KM, Bassett R, Swiler W, Sandford B, Avery S, et al. ED procedural sedation of elderly patients: Is it safe? Am J Emerg Med. 2011;29(5):541-4.

14. Royal College of Anaesthetists and The College of Emergency Medicine. Safe sedation of adults in the emergency department [internet]. 2012. Disponible en: https://www.rcem.ac.uk/docs/College\% 20Guidelines/5z7.\%20Safe\%20Sedation\% 20in\%20the\%20Emergency\%20Departme nt\%20-\%20Report\%20and\%20Recommen dations.pdf

15. Crystal CS, McArthur TJ, Harrison B. Anesthetic and procedural sedation techniques for wound management. Emerg Med Clin North Am. 2007;25(1):41-71.

16. Vinson DR, Hoehn C. Sedationassisted orthopedic reduction in emergency medicine: The safety and success of a one physician/one nurse model. West J Emerg Med. 2013;14(1):47-54.

17. O'Connor RE, Sama A, Burton JH, Callaham ML, House HR, Jaquis WP, et al. Procedural sedation and analgesia in the emergency department: Recommendations for physician credentialing, privileging, and practice. Ann Emerg Med. 2011;58(4):365-70.

18. Josephy CP, Vinson DR. Feasibility of singlevs two-physician procedural sedation in a small community emergency department. Am J Emerg Med. 2018;36(6):977-82.

19. Green SM, Krauss B. Supplemental oxygen during propofol sedation: yes or no? Ann Emerg Med. 2008;52(1):9-10.

20. Mohr NM, Wessman B. Continuous capnography should be used for every emergency department procedural sedation. Ann Emerg Med. 2013 Jun;61(6):697-8.

21. Terp S, Schriger DL. Routine capnographic monitoring is not indicated for all patients undergoing emergency department procedural sedation. Ann Emerg Med. 2013 Jun;61(6):698-9.

22. Green SM, Pershad J. Should capnographic monitoring be standard practice during emergency department procedural sedation and analgesia? Pro and con. Ann Emerg 
Med. 2010 Mar;55(3):265-7. https://doi.or $\mathrm{g} / 10.1016 / \mathrm{j}$.annemergmed.2009.08.019

23. Apfelbaum JL, Gross JB, Connis RT, Agarkar M, Arnold DE, Coté CJ, et al. Practice guidelines for moderate procedural sedation and analgesia 2018. Anesthesiology. 2018 Mar;128(3):437-479. https://doi.org/10.10 97/ALN.0000000000002043

24. Green SM, Roback MG, Miner JR, Burton JH, Krauss B. Fasting and emergency department procedural sedation and analgesia: a consensus-based clinical practice advisory. Ann Emerg Med. 2007;49(4):454-61.

25. Schmitz A, Kellenberger CJ, Neuhaus D, Schroeter E, Deanovic D, Prüfer F, et al. Fasting times and gastric contents volume in children undergoing deep propofol sedation--an assessment using magnetic resonance imaging. Paediatr Anaesth. 2011 Jun;21(6):685-90.

26. Molina JAD, Lobo CA, Goh HK, Seow E, Heng BH. Review of studies and guidelines on fasting and procedural sedation at the emergency department. Int J Evid Based Healthc. 2010 Jun;8(2):75-8.

27. Miner JR, Burton JH. Clinical practice advisory: emergency department procedural sedation with propofol: 2018 update. Ann Emerg Med. 2007;50(2): 182-7. https://doi.org/10.1016/j.annemerg med.2006.12.017

28. Parameters P. practice guidelines for preoperative fasting and the use of pharmacologic agents to reduce the risk of pulmonary aspiration: application to healthy patients undergoing elective procedures. Anesthesiology. 2017;126(3):376-93.

29. Atkinson P, French J, Nice CA. Procedural sedation and analgesia for adults in the emergency department. BMJ. 2014;348(May):1-5.

30. Myers JG, Kelly J. Procedural sedation and analgesia in adults. En: Tintinalli JE, Ma OJ, Yealy DM, Meckler GD, Stapczynski JS, Cline DM, et al., editores. Tintinalli's emergency medicine: a comprehensive study guide. 9. ${ }^{\text {a }}$ ed. Nueva York, NY: McGraw-Hill Education; 2020.

31. Green SM, Mason KP, Krauss BS. Ketamine and propofol sedation by emergency medicine specialists: Mainstream or menace? $\mathrm{Br} \mathrm{J}$ Anaesth. 2016;116(4):449-51.

32. Jalili M, Bahreini M, Doosti-Irani A, Masoomi R, Arbab M, Mirfazaelian H. Ketamine-propofol combination (ketofol) vs propofol for procedural sedation and analgesia: Systematic review and meta-analysis. Am J Emerg Med. 2016;34(3):558-69.

33. Green SM, Roback MG, Kennedy RM, Krauss B. Clinical practice guideline for emergency department ketamine dissociative sedation: 2011 update. Ann Emerg Med. 2011;57(5):449-61.

34. Eich C, Verhagen-Henning S, Roessler M, Cremer F, Cremer S, Strack M, et al. Low-dose S-ketamine added to propofol anesthesia for magnetic resonance imaging in children is safe and ensures faster recovery--a prospective evaluation. Paediatr Anaesth. 2011;21(2):176-8. https://doi.org/10.1111/j $.1460-9592.2010 .03489 . x$

35. Zanos P, Moaddel R, Morris PJ, Riggs LM, Highland JN, Georgiou P, et al. Ketamine and ketamine metabolite pharmacology: insights into therapeutic mechanisms. Pharmacol Rev. 2018 Jul;70(3):621-60.

36. Bell A, Treston G, McNabb C, Monypenny $\mathrm{K}$, Cardwell R. Profiling adverse respiratory events and vomiting when using propofol for emergency department procedural sedation. Emerg Med Australas. 2007 Oct;19(5):405-10. https://doi.org/10.1111/ j.1742-6723.2007.00982.x

37. Bellolio MF, Gilani WI, Barrionuevo P, Murad MH, Erwin PJ, Anderson JR, et al. Incidence of adverse events in adults undergoing procedural sedation in the emergency department: a systematic review and meta-analysis. Acad Emerg Med. 2016;23(2):119-34. 
38. Levins T. Etomidate in procedural sedation. Air Med J. 2011;30(1):45-8.

39. Miner JR, Danahy M, Moch A, Biros M. Randomized clinical trial of etomidate versus propofol for procedural sedation in the emergency department. Ann Emerg Med. 2007 Jan;49(1):15-22.

40. Yates AM, Wolfson AB, Shum L, Kehrl T. A descriptive study of myoclonus associated with etomidate procedural sedation in the ED. Am J Emerg Med. 2013 May;31(5):852-4.

41. Vinca N, Barrett J, Tems CJD. Procedural sedation in the emergency department. En: Out of operating room anesthesia: a comprehensive review. Springer; 2016. p. 305-17. https://doi.org/10.1007/978-3-319. 39150-2_22

42. Çoruh B, Tonelli MR, Park DR. Fentanylinduced chest wall rigidity. Chest. 2013 Apr;143(4):1145-6.

43. Brown TB, Lovato LM, Parker D. Procedural sedation in the acute care setting. Am Fam Physician. 2005;71(1):85-90.

44. Pinto RF, Bhimani M, Milne WK, Nicholson K. Procedural sedation and analgesia in rural and regional emergency departments. Can J Rural Med. 2013;18(4):130-6.

45. Uri O, Behrbalk E, Haim A, Kaufman E, Halpern P. Procedural sedation with propofol for painful orthopaedic manipulation in the emergency department expedites patient management compared with a midazolam/ketamine regimen: a randomized prospective study. J Bone Joint Surg Am. 2011 Dec;93(24):2255-62.

46. Sacchetti A, Senula G, Strickland J, Dubin $R$. procedural sedation in the community emergency department: initial results of the ProSCED Registry. Acad Emerg Med. 2007;14(1):41-6.

47. McGrane O, Hopkins G, Nielson A, Kang C. Procedural sedation with propofol: a retrospective review of the experiences of an emergency medicine residency program 2005 to 2010. Am J Emerg Med. 2012;30(5):706-11.
48. Sury M, Bullock I, Rabar S, Demott K. Guidelines Sedation for diagnostic and therapeutic procedures in children and young people: summary of NICE guidance. BMJ. 2010 Dec 16;341:c6819.

49. Wood-Thompson DK, Enyuma COA, Laher AE. Procedural sedation and analgesia practices in the emergency centre. African J Emerg Med. 2019 Mar;9(1):8-13. https:// doi.org/10.1016/j.afjem.2018.09.003

50. Chawla N, Boateng A, Deshpande R. Procedural sedation in the ICU and emergency department. Curr Opin Anaesthesiol. 2017 Aug;30(4):507-12. https://doi.org/10.1097/ ACO.0000000000000487 